\title{
Primary spinal multifocal intradural-extramedullary Ewing sarcoma in children: presentation of a case and review of the literature
}

\author{
Eren Müngen ${ }^{1 \oplus, ~ N i l g u ̈ n ~ K u r u c u}{ }^{1 \oplus}$, Tezer Kutluk ${ }^{1 \oplus}$, Kader K. Oğuz $^{2 \oplus}$, \\ Figen Söylemezoğlu ${ }^{3 \oplus}$, Bilgehan Yalçın ${ }^{1 \oplus}$ \\ ${ }^{1}$ Departments of Pediatric Oncology, Hacettepe University Faculty of Medicine and Cancer Institute Ankara; Departments of \\ ${ }^{2}$ Radiology and ${ }^{3}$ Pathology, Hacettepe University Faculty of Medicine, Ankara, Turkey.
}

\begin{abstract}
Background. Primary spinal, intradural, extramedullary Ewing sarcoma (PSIEES) is exceptionally uncommon. Here, we present an interesting pediatric case with a PSIEES diagnosis confirmed by the presence of a specific fusion protein in the tumor tissue and who then developed a cerebellar recurrence. We also reviewed the PSIEES cases in childhood reported in the literature.

Case. An 8.5-year-old boy was admitted to a local hospital with a one-month history of severe back and limb pain, and inability to move his lower limbs. Physical examination revealed paraparesis in the lower extremities. Spinal MRI revealed multiple intradural extramedullary masses at the L2-L3, L4-5 and L5-S1 levels. He underwent surgery and near total excision of all three masses were performed. Histopathological diagnosis of Ewing Sarcoma was confirmed with EWS-ERG gene rearrangement. The patient was treated according to EuroEwing chemotherapy protocol. A total dose of 4500 cGy radiotherapy was applied to the tumor location at L2-S1 paravertebral region. Eighteen months after the end of treatment, a mass in the left cerebellar hemisphere was determined. Gross total excision was performed. Histopathological examination of the tumor showed Ewing sarcoma. Radiological screening revealed isolated central nervous system recurrence. A total of $4500 \mathrm{cGy}$ radiotherapy was applied. He is on a second-line treatment consisting of gemcitabine and docetaxel without any evidence of disease.

Conclusions. Ewing Sarcoma with spinal intradural region in childhood is very rare. We could only find 17 pediatric cases reported in the literature. Neurological findings occur earlier in tumors of this region. The prognosis is worse than other extraosseous Ewing sarcoma.
\end{abstract}

Key words: Ewing family of tumors, extraosseous Ewing sarcoma, intradural extramedullary tumors, cord compression.

Ewing's sarcoma (ES) family of tumors including Ewing sarcoma of bone, extraosseous Ewing sarcoma (EOES), primitive neuroectodermal tumors (PNET), Ewing sarcoma of the chest wall (Askin tumor) and atypical ES are highly aggressive mesenchymal neoplasms which originate from mesenchymal progenitor cells and which share specific chromosomal

$\triangle$ Eren Müngen

mungeneren@gmail.com

Received 17th March 2021, revised 3th May 2021, accepted 13th May 2021. translocation $(21 ; 22)(\mathrm{q} 22 ; \mathrm{q} 12)$ resulting in a fusion of EWSR1 gene product with a member of the ETS family of transcription factors. ${ }^{1}$ Ewing's sarcoma family of tumors account for $1.5-2 \%$ all childhood cancers. Ewing sarcoma of bone is the second most common malignant bone tumor in childhood that arises from long, short or flat bones. ${ }^{1,2}$ In children, primary extraosseous manifestation has been reported in $15-20 \%$ of cases while approximately half of the cases are located in the extraskeletal regions in adults. ${ }^{3}$ Extraosseous ES is more frequently seen in adolescent males. It is 
more likely to originate from soft tissue of the thorax and abdomen (43\%), extremities $(26 \%)$ and pelvis $(14 \%)$. The paravertebral region is a relatively rare location for EOES and constitutes approximately $7 \%$ of them. ${ }^{4}$ In paravertebral space, tumors almost always arise from extradural paraspinal soft tissue. Primary spinal intradural-extramedullary ES family of tumor (PSIEES) is extremely rare. ${ }^{4,5}$

In this study, we present a rare pediatric case with PSIEES-confirmed with its characteristic translocation of EWS-ERG and relapsed disease in cerebellum which make this case very interesting. We also reviewed the pediatric PSIEES cases reported in the literature.

\section{Case Report}

An 8.5-year-old previously healthy boy was admitted to a local hospital with one month history of severe back and limb pain, and inability to move his lower limbs for the last few days. Physical examination revealed paraparesis in the lower extremities. Spinal MRI revealed multiple intradural extramedullary masses homogenously enhancing with intravenously administered Gadolinium-based contrast material at the L2-L3 level (3.8x1.6x1.2 $\mathrm{cm})$, L4-5 level $(2.6 \times 0.9 \times 1.1 \mathrm{~cm})$ and L5-S1 level $(1.1 \times 0.4 \times 0.7 \mathrm{~cm})$ (Fig. 1). Metastatic workup with thoracic CT and PET-CT were performed for metastatic screening and found to be normal. He underwent surgery and near total excision of all three masses with L4-7 laminectomy was performed in the local hospital. After surgery, his symptoms improved and he was referred to our clinic.

Histopathologically, the tumor was composed of uniform small round cells with round nuclei containing fine chromatin and scanty eosinophilic cytoplasm. Neoplastic cells were found strongly positive for CD99, patchy and moderately positive for synaptophysin. These cells were negative for GFAP, NSE, inhibin, PanCK, EMA, desmin, LCA, S100 and WT1. The $\mathrm{Ki}-67$ proliferation index was greater than $90 \%$. Loss of INI1 expression was not detected. In
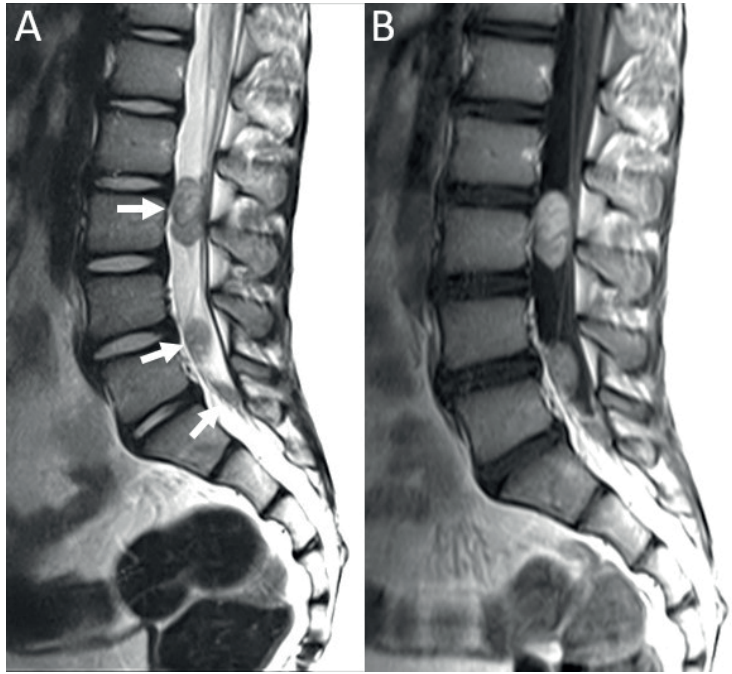

Fig. 1. Lumbar spinal MRI shows multiple intradural masses, which are T2 isointense to the spinal cord (arrows, sagittal T2-weighted image, A) and enhancing homogenously following gadolinium based contrast material administration (sagittal T1weighted postcontrast image).

tumor tissue with RT-PCR, the fusion product related to EWS-ERG gene rearrangement was detected.

The patient was treated according to EuroEwing protocol. After six courses of vinkristin, ifosfamide, doxorubicin and etoposide (VIDE) treatment, a total dose of 4500 cGy radiotherapy was applied to the tumor location at L2-S1 paravertebral region. Then he received six cycles of VAC regimen (vincristine, actinomycin-D and cyclophosphamide). After the end of treatment the patient remained in remission for 18 months.

Three years after his first diagnosis, the patient came back with persistent headache for two weeks. Cranial MRI revealed a mass $(4 \times 4.5 \mathrm{~cm})$ consisting of cystic and solid components in the left cerebellar hemisphere (Fig. 2). Spinal MRI was normal. Radiological screening revealed that it was isolated central nervous system recurrence. Gross total excision of the mass was performed.

The histopathological findings of the cerebellar mass were identical to those of the previous 


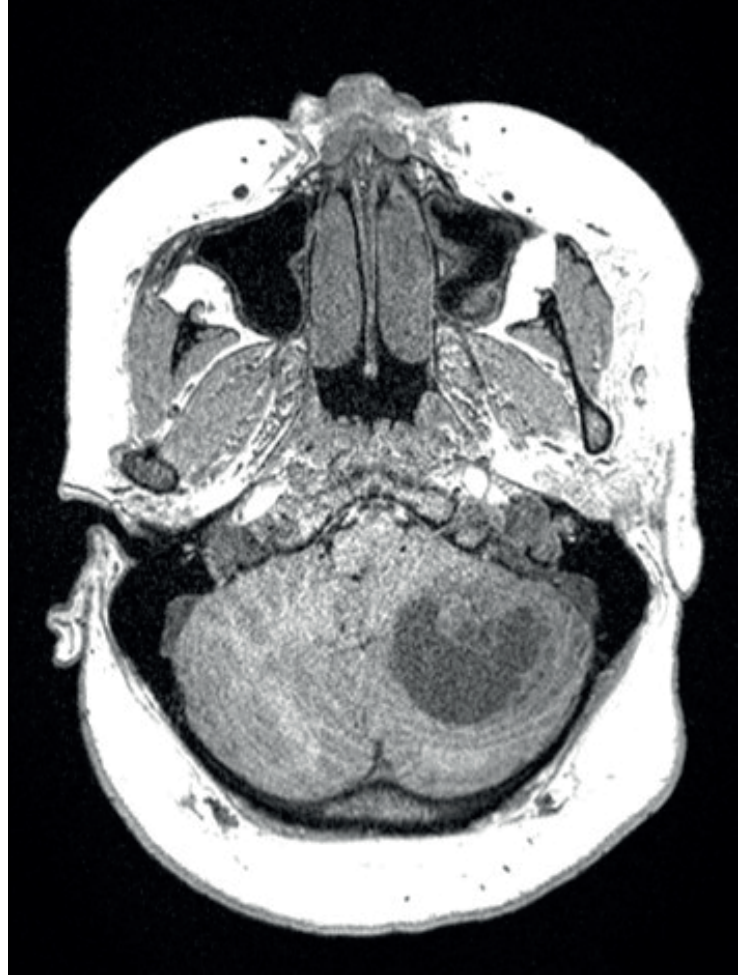

Fig. 2. Axial T1-weighted image shows a cystic mass with solid component in the left cerebellar hemisphere compressing the brainstem.

spinal intradural mass. The histopathological diagnosis of Ewing sarcoma was confirmed again with the presence of EWS-ERG fusion.

A total of 4500 cGy radiotherapy was applied to the cerebellar mass. He is on a secondline treatment consisting of gemcitabine and docetaxel without any evidence of disease.

The parents and patient were informed about the purpose of the case report presentation and informed consent was obtained.

\section{Discussion}

Childhood tumors located in the perispinal region present with symptoms of spinal cord compression. In addition to intradural tumors, extradural paraspinal tumors may also cause spinal cord compression by extending through the neural foramina to the epidural region. In childhood, most common extradural tumors are neuroblastoma, ES and soft tissue sarcomas.
Intradural extramedullary tumors consist of meningiomas, neurofibromas, schwannomas and malignant peripheral nerve sheath tumors. Ewing sarcomas causing spinal cord compression arise either from vertebrae or the extradural paravertebral region. The clinical possibility of ES is extremely low for intradural extramedullary tumors. ${ }^{6,7}$ Ewing sarcoma rarely originates from the intradural region and constitutes only $2,9 \%$ of all perispinal ES in adults. ${ }^{4}$ Less than 50 cases with PSIEES have been reported in the literature. ${ }^{5}$

In the literature, we could find only 17 pediatric cases $(<18$ years old $)$ with primary spinal intradural ES family of tumors., ${ }^{4-20}$ Histopathological diagnosis were EOES in ten and PNET in seven. We analyzed the clinical features of 18 cases in total, including our case (Table I). There were twelve boys and six girls $(\mathrm{M} / \mathrm{F}=2)$. Their ages ranged from 5 to 17 years old with a median age of 14 years. The duration of complaints ranged from 20 days to one year. The most common complaints were back pain (n:7), leg pain (n:2), back and extremity pain (n:3), and motor weakness (n:5). Physical examination findings were not reported in four of the 18 cases. Among the remaining 14, loss of strength in the limbs was found in seven cases, sensory loss at limbs in eight cases, and reflex changes at lower extremities in one case. In seventeen of the 18 cases, there was a single mass which was most frequently located in the lumbosacral and thoracic regions, respectively. Interestingly, our case had three separate masses close to each other. None of the reviewed cases including ours had metastasis at diagnosis.

Molecular study was reported in four of 18 patients. EWSR1-FLI1 was found in two patients and EWS-ERG fusion transcripts were found in our case. In the fourth case the type of the translocation was not given. Gross total or subtotal resection was performed in all cases. There was no information about chemotherapy and radiotherapy in third and fourth cases, respectively. Nine cases received chemotherapy and eight received radiotherapy. One case died within one month after the operation. Thirteen 


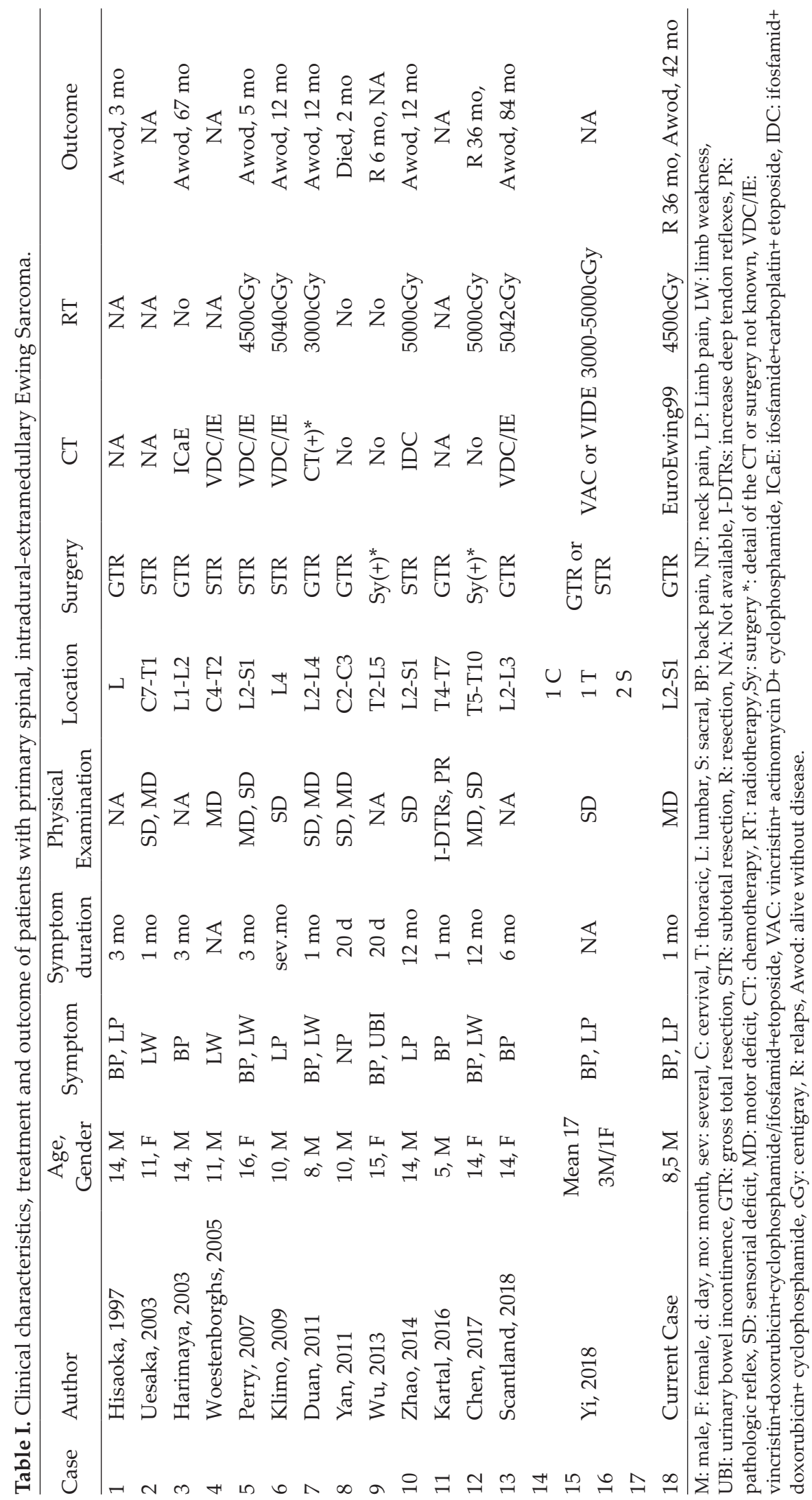


cases were alive without disease at a median of 12 (3-27) months. Two of 17 cases reviewed had relapsed disease at 6 and 36 months, respectively. Relapse site was not reported at the first case while local and systemic (lymph nodes, bones, bone marrow and leptomeninges) relapse was observed in the second case. Our case was also alive without disease at 42 months after a cerebellar relapse at 36 months after diagnosis, and his treatment is continuing.

There are some striking differences between ESB, EOES and PSIEES. When we consider the results of two major studies that compare the clinical features of ESB and EOES, there are no meaningful differences in age or sex between these two groups. In childhood both are seen more frequently in adolescence and slightly more in males. ${ }^{21,22}$ In contrast, PSIEES is seen at a younger age (median 11 years) with an obvious male preponderance $(\mathrm{M} / \mathrm{F}=4)$.

Clinical characteristics and outcome are different in ESB and EOES. Tumors are more likely to be smaller than $8 \mathrm{~cm}$ and more commonly arise from axial locations in ESB. There were no differences in metastatic status between ESB and EOES. In both groups 30\% of patients had metastatic disease. In ES located in the paraspinal space, extra or intradural regions, the close proximity of the tumoral mass to the adjacent neural elements leads to early neurological symptoms. Thus, diagnosis usually takes place before the tumor reaches a large volume, and before the systematic spread of the disease. ${ }^{23}$ Brain metastasis has been reported in $8-10 \%$ of cases in various series involving ESB while in paravertebral extradural ES, this was $3.3 \%{ }^{24,25}$ In cases with PSIEES, central nervous system metastasis was not reported except for a few cases with medulla spinalis metastasis. ${ }^{26,27}$ The majority of brain metastases in ES present with a single mass generally located in the parietal or frontal lobe, or with diffuse multiple nodules..$^{24,25}$ We could not find any reported cases with ES metastasis to the cerebellum at diagnosis or at relapse in English literature.

The management of ES requires a multidisciplinary approach, including surgical resection, adjuvant chemotherapy, and radiotherapy. ${ }^{4,16,23}$ Complete resection of the tumor has been reported to have a positive effect on prognosis and survival. However, due to the location of the tumor near the spinal canal and its close proximity to the adjacent neural elements, gross total tumor resection may not always be possible. Furthermore, it may not be possible to ensure that the tumor is not disseminated through the cerebrospinal fluid after an aggressive surgery. Chemotherapy and radiotherapy appear to be main treatment modalities in PSIEES. ${ }^{13,19}$ In our case, despite the combination of surgical treatment, chemotherapy and radiotherapy, brain metastasis appeared after a while.

Although morphological and genomic features of EOES are indistinguishable from classical ESB, a better outcome has been reported in EOES than that of ESB. ${ }^{5}$ Both overall and event free survival are significantly better in EOES than ESB ( $85 \%$ vs $78 \%$ for OS and $76 \%$ vs $69 \%$ for EFS). However, prognosis of EOES located in the perispinal region is worse than in other extraosseous locations. It was reported in adult cases with PSIEES that the OS rate was $40 \%$ with a median 14 months and the overall recurrence rate was $46 \% .{ }^{21,22}$ In pediatric PSIEES, only nine of 18 reviewed cases were alive without disease at a median of 12 months. Although recurrence was reported in only three of the cases, it must be considered that the follow up time was short.

The spinal intradural region is an extremely uncommon location for ES in children. Although early diagnosis is often possible thanks to the early appearance of neurological symptoms due to proximity of the tumor to the spinal cord, the outcome of the patients tends to be worse than ES of other sites.

\section{Author contribution}

The authors confirm contribution to the paper as follows: study conception and design: EM, $\mathrm{NK}$; data collection: $\mathrm{EM}, \mathrm{KKO}, \mathrm{FS}$; analysis and interpretation of results: $\mathrm{EM}, \mathrm{NK}, \mathrm{TK}, \mathrm{KKO}, \mathrm{FS}$, 
BY; draft manuscript preparation: EM, NK, TK. All authors reviewed the results and approved the final version of the manuscript.

\section{Conflict of interest}

The authors declare no conflict of interest.

\section{REFERENCES}

1. Riggi N, Stamenkovic I. The Biology of Ewing sarcoma. Cancer Lett 2007; 254: 1-10. https://doi. org/10.1016/j.canlet.2006.12.009

2. Bernstein M, Kovar H, Paulussen M, et al. Ewing's sarcoma family of tumors: current management. Oncologist 2006; 11: 503-519. https://doi.org/10.1634/ theoncologist.11-5-503

3. Javery O, Krajewski K, O'Regan $\mathrm{K}$, et al. A to Z of extraskeletal Ewing sarcoma family of tumors in adults: imaging features of primary disease, metastatic patterns, and treatment responses. AJR Am J Roentgenol 2011; 197: W1015-W1022. https:// doi.org/10.2214/AJR.11.6667

4. Yan Y, Xu T, Chen J, Hu G, Lu Y. Intraspinal Ewing's sarcoma/primitive neuroectodermal tumors. J Clin Neurosci 2011; 18: 601-606. https://doi.org/10.1016/j. jocn.2010.09.012

5. Lu VM, Goyal A, Alvi MA, Kerezoudis P, Haddock MG, Bydon M. Primary intradural Ewing's sarcoma of the spine: a systematic review of the literature. Clin Neurol Neurosurg 2019; 177: 12-19. https://doi. org/10.1016/j.clineuro.2018.12.011

6. Pollono D, Tomarchia S, Drut R, Ibanez O, Ferreyra $\mathrm{M}$, Cedola J. Spinal cord compression: a review of 70 pediatric patients. Pediatr Hematol Oncol 2003; 20: 457-466. https://doi.org/10.1080/08880010390220126

7. Koeller KK, Shih RY. Intradural extramedullary spinal neoplasms: radiologic-pathologic correlation. Radiographics 2019; 39: 468-490. https://doi. org/10.1148/rg.2019180200

8. Hisaoka M, Hashimoto $H$, Murao T. Peripheral primitive neuroectodermal tumour with ganglioneuroma-like areas arising in the cauda equina. Virchows Arch 1997; 431: 365-369. https:// doi.org/10.1007/s004280050112

9. Uesaka T, Amano T, Inamura T, et al. Intradural, extramedullary spinal Ewing's sarcoma in childhood. J Clin Neurosci 2003; 10: 122-125. https:// doi.org/10.1016/S0967-5868(02)00279-5
10. Harimaya K, Oda Y, Matsuda S, Tanaka K, Chuman $\mathrm{H}$, Iwamoto Y. Primitive neuroectodermal tumor and extraskeletal Ewing sarcoma arising primarily around the spinal column: report of four cases and a review of the literature. Spine (Phila Pa 1976) 2003; 28: E408-E412. https://doi.org/10.1097/01. BRS.0000085099.47800.DF

11. Woestenborghs H, Debiec-Rychter M, Renard M, et al. Cytokeratin-positive meningeal peripheral PNET/Ewing's sarcoma of the cervical spinal cord: diagnostic value of genetic analysis. Int J Surg Pathol 2005; 13: 93-97. https://doi. org/10.1177/106689690501300114

12. Perry R, Gonzales I, Finlay J, Zacharoulis S. Primary peripheral primitive neuroectodermal tumors of the spinal cord: report of two cases and review of the literature. J Neurooncol 2007; 81: 259-264. https:// doi.org/10.1007/s11060-006-9178-1

13. Klimo P Jr, Codd PJ, Grier H, Goumnerova LC Primary pediatric intraspinal sarcomas. Report of 3 cases. J Neurosurg Pediatr 2009; 4: 222-229. https:// doi.org/10.3171/2009.3.PEDS08272

14. Duan $X H$, Ban $X H$, Liu B, et al. Intraspinal primitive neuroectodermal tumor: imaging findings in six cases. Eur J Radiol. 2011; 80: 426-431. https://doi. org/10.1016/j.ejrad.2010.06.005

15. Wu G, Ghimire P, Zhu L, Baral A, Su J. Magnetic resonance imaging characteristics of primary intraspinal peripheral primitive neuroectodermal tumour. Can Assoc Radiol J 2013; 64: 240-245. https:// doi.org/10.1016/j.carj.2012.01.001

16. Zhao M, Zhang B, Liang F, Zhang J. Primary spinal intradural extraskeletal Ewing sarcoma mimicking a giant nerve sheath tumor: case report and review of the literature. Int J Clin Exp Pathol 2014; 7: 90819085.

17. Kartal A, Akatli A. Primary intradural extraosseous Ewing's sarcoma in a young child. Childs Nerv Syst 2016; 32: 409-410. https://doi.org/10.1007/s00381-0163020-7

18. Chen F, Chiou SS, Lin SF, Lieu AS, Chen YT, Huang CJ. Recurrent spinal primitive neuroectodermal tumor with brain and bone metastases: a case report. Medicine (Baltimore) 2017; 96: e8658. https://doi. org/10.1097/MD.0000000000008658

19. Scantland JT, Gondim MJ, Koivuniemi AS, Fulkerson DH, Shih CS. Primary spinal intradural extraosseous Ewing sarcoma in a pediatric patient: case report and review of the literature. Pediatr Neurosurg 2018; 53: 222-228. https://doi.org/10.1159/000488767 
20. Yi X, Zhou M, Liao S, et al. Spinal peripheral primitive neuroectodermal tumors: a radiological analysis of ten cases. Turk Neurosurg 2019; 29: 3342. https://doi.org/10.5137/1019-5149.JTN.21931-17.2

21. Applebaum MA, Worch J, Matthay KK, et al. Clinical features and outcomes in patients with extraskeletal Ewing sarcoma. Cancer 2011; 117: 3027-3032. https:// doi.org/10.1002/cncr.25840

22. Cash T, Mcllvaine E, Krailo MD, et al. Comparison of clinical features and outcomes in patients with extraskeletal versus skeletal localized Ewing sarcoma: a report from the Children's Oncology Group. Pediatr Blood Cancer 2016; 63: 1771-1779. https://doi.org/10.1002/pbc.26096

23. Paterakis KN, Brotis A, Dardiotis E, Giannis T, Tzerefos C, Fountas KN. Multimodality treatment of intradural extramedullary Ewing's sarcomas. A systematic review. Clin Neurol Neurosurg 2018; 164: 169-181. https://doi.org/10.1016/j. clineuro.2017.11.014
24. Shuper A, Cohen IJ, Mor C, Ash S, Kornreich L, Zaizov R. Metastatic brain involvement in Ewing family of tumors in children. Neurology 1998; 51: 1336-1338. https://doi.org/10.1212/WNL.51.5.1336

25. Parasuraman S, Langston J, Rao BN, et al. Brain metastases in pediatric Ewing sarcoma and rhabdomyosarcoma: the St. Jude Children's Research Hospital experience. J Pediatr Hematol Oncol 1999; 21: 370-377. https://doi.org/10.1097/00043426199909000-00007

26. Haresh KP, Chinikkatti SK, Prabhakar R, et al. A rare case of intradural extramedullary Ewing's sarcoma with skip metastasis in the spine. Spinal Cord 2008; 46: 582-584. https://doi.org/10.1038/sc.2008.8

27. Gong HS, Huang QS, Liu GJ, Chen FH, Zhao HB. Cervical primary Ewing's sarcoma in intradural and extramedullary location and skip metastasis to cauda equina. Turk Neurosurg 2015; 25: 943-947. https://doi.org/10.5137/1019-5149.JTN.12025-14.2 